\title{
Análise do perfil epidemiológico da Doença de Chagas no Brasil. Período entre 2001 e 2018
}

\section{Analysis of the epidemiological profile of Chagas disease in Brazil. Period between 2001 and 2018}

Thaís Lemos de Souza Macêdo ${ }^{1 *}$, Sara Cristine Marques dos $\operatorname{Santos}^{1}$, Renata Baptista dos Reis Rosa ${ }^{2}$, Pietra Moreira Vieira $^{2}$, Caio Teixeira dos Santos ${ }^{1}$, Ivana Picone Borges de Aragão ${ }^{3}$

Como citar esse artigo. Macêdo,

T.L.S.; dos Santos, S.C.M.; Rosa,

R.B.R.; Vieira, P.M.; dos Santos, C.T.; de Aragão, I.P.B. Análise do perfil epidemiológico da Doença de Chagas no Brasil. Período entre 2001 e 2018 Revista de Saúde 2021 Ago./Nov.; $12 \quad$ (3): $\quad 42-49$.

\section{Resumo}

A Doença de Chagas (DC) é considerada uma antropozoonose de ocorrência em região tropical, causada pelo protozoário flagelado Trypanosoma cruzi. Trata-se de uma doença negligenciada, onde existem cerca de 8 milhões de indivíduos infectados, sendo $28 \mathrm{mil} \mathrm{casos/ano} \mathrm{de} \mathrm{registros} \mathrm{de} \mathrm{incidência} \mathrm{e} 12$ mil óbitos/ano. No Brasil, foram registrados 1.156 .821 casos no ano de 2010. O objetivo deste estudo foi avaliar o perfil epidemiológico dos pacientes diagnosticados com DC aguda e a prevalência brasileira da doença nas últimas duas décadas. Foi realizado, estudo documental, retrospectivo, e histórico, a partir da extração de dados do Departamento de Informática do Sistema Único de Saúde - DATASUS, oriundos do formulário "Sistema de Informação de Agravos de Notificação" (SINAN), entre o período de 2001 a 2018 , e dividido em duas fases de análise: primeira década (2001-2010) e segunda década (2011-2018), possibilitando comparações entre os subperíodos temporais. Durante o período analisado, 5.184 notificações de DC aguda (288 pacientes/ano) foram registradas, sendo a região Norte a mais frequente do país. O sexo masculino, pardo e adultos jovens são os perfis mais prevalente entre os infectados. A variabilidade dos dados de prevalência da doença em demais estudos demonstra a limitação dos achados epidemiológicos e a presença de um silêncio epidemiológico. Acredita-se que esse dado seja muito maior na realidade da prática médica, devido aos casos de subnotificações. Dessa forma, se mostra necessária medidas preventivas e de monitorização epidemiológica dos casos confirmados e suspeitos, afim de que haja controle de possíveis focos vetoriais e outras formas de infecção.

Palavras-chave: Doença de Chagas, Trypanosoma cruzi, Tripanossomíase, Epidemiologia, Saúde Pública.

\begin{abstract}
Chagas disease (CD) is considered an anthropozoonosis occurring in a tropical region, caused by the flagellated protozoan Trypanosoma cruzi. It is a neglected disease, where there are about 8 million infected individuals, 28 thousand cases/ year of incidence records, and 12 thousand deaths/year. In Brazil, 1,156,821 cases were recorded in 2010. The objective this study was to assess the epidemiological profile of patients diagnosed with acute $\mathrm{CD}$ and the Brazilian prevalence of the disease in the last two decades. Was performed, a documentary, retrospective, historical study, based on data extraction from the Informatics Department of the Unified Health System - DATASUS, from the form "Information System for Notifiable Diseases" (SINAN), between the period 2001 to 2018, and divided into two phases of analysis: first decade (2001-2010) and second decade (2011-2018), allowing comparisons between temporal subperiods. During the period analyzed, 5,184 notifications of acute CD (288 patients/year) were recorded, with the North region being the most frequent in the country. Males, browns, and young adults are the most prevalent profiles among those infected. The variability of data on the prevalence of the disease in other studies demonstrates the limitation of the findings and the presence of an epidemiological silence. It is believed that this figure is much higher in the medical practice reality, due to cases of underreporting. Thus, preventive measures and epidemiological monitoring of confirmed and suspected cases are necessary, to control possible vector outbreaks and other forms of infection.
\end{abstract}

Keywords: Chagas Disease, Trypanosoma cruzi, Trypanosomiasis, Epidemiology, Public Health.

\section{Introdução}

A Doença de Chagas (DC) ou tripanossomíase americana ocorre em região tropical sendo considerada como uma antropozoonose, causada pelo protozoário flagelado Trypanosoma cruzi (T. cruzi) e transmitida através do inseto triatomíneo, do gênero, triatoma, conhecido como barbeiro. O T. cruzi possui ampla distribuição geográfica no ocidente, primariamente com ocorrências apenas nas Américas, em virtude do vetor está restrito ao continente. Há relatos de contaminação em até 21 países das Américas, desde os Estados Unidos até América do Sul. Entretanto, são registrados casos em países não endêmicos por outros mecanismos de transmissão (que não a do vetor-hospedeiro) ${ }^{1-3} \mathrm{ou}$ pela migração intensa de latino-americanos para demais continentes ${ }^{4}$.

Descrita em 1909, em Lassance, interior de Minas Gerais, por Carlos Ribeiro Justiniano das Chagas, médico e pesquisador brasileiro, o qual não apenas descobriu a 
nova doença tropical, mas também entrou para a história da medicina mundial, como o primeiro pesquisador a ser responsável por identificar e descrever o vetor, o agente etiológico e a doença causada pelo parasita. Desde então, temos conhecimento do mecanismo de transmissão clássico da $\mathrm{DC}$, que ocorre através do inseto da subfamília Triatominae, que ao realizar um novo repasto sanguíneo em um indivíduo saudável, elimina nas fezes T. cruzi, em forma de tripomastigotas metacíclicos, que penetram na ferida da picada. Dentro das células hospedeiras dos vertebrados, elas se tornam amastigotas e se multiplicam, formando ninhos intracelulares que se diferenciam em tripomastigota e é nessa forma que há liberação na corrente sanguínea para atingir outros tecidos ou órgãos ${ }^{5}$.

A DC pode se manifestar de forma aguda ou crônica, seja nas formas indeterminadas, cardíaca, digestiva ou cardiodigestiva. Cerca de 20 a $30 \%$ dos indivíduos infectados irão desenvolver cardiomiopatia e/ou síndromes digestivas, levando a complicações ou óbito, com repercussões sociais e econômicas ${ }^{6}$.

É classificada como uma das 17 doenças tropicais negligenciadas segundo a Organização Mundial de Saúde $(\mathrm{OMS})^{7,8}$. A incidência da DC tem relação com o nível socioeconômico da região, estudos comprovam que a maior concentração de população em áreas urbanas e a precariedade das condições de habitação, saneamento e renda são considerados como determinantes e condicionantes sociais para a transmissão de T. cruzi ${ }^{5}$, 9-12.

Segundo a OMS, existem cerca de 8 milhões de indivíduos infectados por T. cruzi em nível mundial, sendo 28 mil casos por ano de registros de incidência da doença e cerca de 12 mil óbitos por ano ${ }^{13}$. A estimativa dos registros de 2010, demonstrou que nos 21 países latino-americanos, havia 5.742.167 pessoas infectadas por $T$. cruzi, sendo que 3.581 .423 destas $(62,4 \%)$ se encontravam nos países daAmérica do Sul, com destaque à Argentina (1.505.235), ao Brasil (1.156.821) e Bolívia $(607.186)^{14}$. Esses dados evidenciam a expressividade da infecção no contexto social da América do Sul, principalmente, no Brasil ${ }^{15}$.

Em contrapartida, os dados nos países não endêmicos mostram registros menos expressivos. Em 2005, estimou-se um total de 501.036 brasileiros nos Estados Unidos da América (EUA), com uma prevalência de infecção por T. cruzi de $1,02 \%$ ou 5.106 casos $^{16-17}$. De uma forma mais ampla, estudos estimam que a quantidade de infectados por T. cruzi nos EUA varie entre 300 mil a 1 milhão de pessoas, ${ }^{18-19}$ repercutindo com diferentes impactos para o sistema de saúde. Já no continente europeu, a estimativa é de que haja cerca de 72 mil pessoas infectadas pelo protozoário ${ }^{20}$.

Segundo o estudo que investigou a prevalência de DC em latino-americanos que vivem na Europa (Espanha, França, Suíça, Itália e Alemanha), foi observado que a prevalência da doença foi de $0,6 \%{ }^{21}$; em 18,1\% em migrantes oriundos da Bolívia; 5,5\% paraguaios; $2,2 \%$ em argentinos e não houve indivíduos detectados entre migrantes do Uruguai, Venezuela, Panamá, Guatemala e México ${ }^{21}$.

O objetivo do trabalho foi avaliar as características epidemiológicas dos pacientes diagnosticados com DC aguda e a prevalência da doença em todo o território brasileiro durante o período de duas décadas.

\section{Material e Métodos}

Foi realizado um estudo documental, retrospectivo e histórico, a partir da coleta de dados de DC aguda entre o período de 2001 a 2018. Os dados epidemiológicos foram obtidos no banco de dados do Departamento de Informática do Sistema Único de Saúde - DATASUS, oriundos do formulário "Sistema de Informação de Agravos de Notificação" (SINAN), documento preenchido pelo médico responsável no momento da internação hospitalar.

O período estudado foi dividido em duas fases de análise para que fosse possível realizar comparações entre os subperíodos temporais de acordo com cada década. Os seguimentos foram denominados como "primeira década", representando o intervalo de anos entre 2001 e 2010, e "segunda década" representando o intervalo de anos entre 2011 a 2018.

Foram incluídos os dados referentes a incidência da doença, sexo, faixa etária, raça, método diagnóstico, local provável de infecção, Unidade Federativa (UF) de infecção e de notificação.

A partir da coleta foi procedida a análise dos dados, estabelecendo o perfil em relação a ocorrência da doença e fomentar possíveis explicações para as variações estatísticas de notificação.

O estudo foi baseado somente nos casos de DC aguda, pois apenas a forma aguda da doença é de notificação compulsória no país, por meio do SINAN, não tendo registros da forma crônica nos bancos de dados do DATASUS.

\section{Resultados}

Entre 2001 e 2018, houve 5.184 notificações de pacientes infectados com DC aguda nas unidades de saúde de todo o território brasileiro.

A primeira década avaliada, entre 2001 e 2010 , demonstrou maior prevalência, com 2.884 (55,7\%) casos e a segunda década, correspondente ao período entre 2011 e 2018, com 2.070 (44,3\%) de casos, sendo que 230 casos da amostra total não foram especificados no DATASUS em qual ano entre o período das duas décadas foram registradas as notificações. Calculado 
uma frequência total de 288 pacientes/ano, sendo o ano de maior registro, o de 2003.

No total, houve notificação de 2.607 (50,28\%) casos em indivíduos do sexo masculino em comparação a $2.575(49,67 \%)$ do sexo feminino. Quando realizada a comparação por décadas, foi possível observar uma mudança de perfil dos infectados ao longo dos 18 anos, uma vez que na primeira década, a incidência registrada foi maior nos indivíduos do sexo feminino, com 1.611 $(55,8 \%)$ casos, enquanto na segunda década, o sexo masculino registrou maior número de doentes, com $1.134(54,8 \%)$ casos (Tabela 1$)$.

A faixa de idade predominante, durante todo período analisado, foi entre 40 e 59 anos, seguido pela de 20 a 39 anos (Tabela 2). Na primeira década houve

Tabela 1. Características dos pacientes diagnosticados com Doença de Chagas aguda, segundo a divisão de primeira e segunda décadas.

\begin{tabular}{|c|c|c|}
\hline Variável & $\begin{array}{c}1^{\circ} \text { década } \\
(2001-2010)\end{array}$ & $\begin{array}{c}2^{\circ} \text { década } \\
(2011-2018)\end{array}$ \\
\hline Sexo & $\mathbf{n}$ & n \\
\hline Masculino & 1473 & 1134 \\
\hline Feminino & 1611 & 964 \\
\hline Ignorado/Branco & 2 & 0 \\
\hline Total & 3086 & 2098 \\
\hline Faixa etária & $\mathbf{n}$ & $\mathbf{n}$ \\
\hline$<1$ ano & 37 & 33 \\
\hline 1 a 4 & 49 & 94 \\
\hline 5 a 9 & 93 & 163 \\
\hline 10 a 14 & 101 & 177 \\
\hline 15 a 19 & 122 & 172 \\
\hline 20 a 39 & 954 & 700 \\
\hline 40 a 59 & 1234 & 511 \\
\hline 60 a 64 & 173 & 92 \\
\hline 65 a 69 & 124 & 54 \\
\hline 70 a 79 & 141 & 76 \\
\hline$>80$ & 54 & 26 \\
\hline Ignorado/Branco & 4 & 0 \\
\hline Total & 3086 & 2098 \\
\hline Região de notificação & $\mathbf{n}$ & $\mathbf{n}$ \\
\hline Norte & 880 & 2003 \\
\hline Nordeste & 1602 & 71 \\
\hline Sudeste & 217 & 8 \\
\hline Sul & 288 & 3 \\
\hline Centro-Oeste & 99 & 13 \\
\hline Total & 3086 & 2098 \\
\hline Cor & $\mathbf{n}$ & $\mathbf{n}$ \\
\hline Ignorado/Branco & 358 & 144 \\
\hline Branca & 847 & 226 \\
\hline Preta & 282 & 94 \\
\hline Amarela & 32 & 9 \\
\hline Parda & 1532 & 1609 \\
\hline Indígena & 35 & 16 \\
\hline Total & 3086 & 2098 \\
\hline Evolução clínica & $\mathbf{n}$ & $\mathbf{n}$ \\
\hline Ignorado/Branco & 1293 & 241 \\
\hline Vivo & 1687 & 1823 \\
\hline $\begin{array}{l}\text { Óbito pelo agravo } \\
\text { notificado }\end{array}$ & 104 & 30 \\
\hline Óbito por outra causa & 2 & 4 \\
\hline Total & 3086 & 2098 \\
\hline
\end{tabular}

predomínio de casos na faixa etária entre 40-59 anos e na segunda década, entre os 20-39 anos (Tabela 1).

A cor predominante foi a parda, com 3.141 casos $(60,59 \%)$ quando avaliado todo o período estudado (Tabela 3).

Os 5.184 casos notificados com DC aguda foram analisados segundo a região brasileira, sendo identificados: $2.883(55,61 \%)$ na região Norte; 1.673 (32,27\%) Nordeste; 291 (5,61\%) Sul; 225 (4,34\%) Sudeste e 112 (2,16\%) do Centro-Oeste.

A comparação por décadas demonstrou uma mudança de perfil da região de maior notificação de DC, ao longo dos dois períodos avaliados. No Nordeste, houve maior notificação na primeira década, com 1.602 casos e no Norte, houve maior destaque na segunda

Tabela 2. Distribuição do número de casos da Doença de Chagas aguda, segundo faixa etária, no Brasil, entre 2001 e 2018 .

\begin{tabular}{cc}
\hline Faixa etária & n \\
\hline Menos de 1 ano & 70 \\
1 a 4 anos & 143 \\
5 a 9 anos & 256 \\
10 a 14 anos & 278 \\
15 a 19 anos & 294 \\
20 a 39 anos & 1.654 \\
40 a 59 anos & 1.745 \\
60 a 64 anos & 265 \\
65 a 69 anos & 178 \\
70 a 79 anos & 217 \\
Mais de 80 anos & 80 \\
Ignorado/Branco & 4 \\
\hline Total & $\mathbf{5 . 1 8 4}$ \\
\hline
\end{tabular}

Tabela 3. Número de casos de Doença de Chagas aguda, segundo a cor, entre 2001 e 2018, no Brasil.

\begin{tabular}{lc}
\hline Cor & $\mathbf{n}$ \\
\hline Branca & 1.073 \\
Ignorado/Branco & 502 \\
Preta & 376 \\
Amarela & 41 \\
Parda & 3.141 \\
Indígena & 51 \\
\hline Total & $\mathbf{5 . 1 8 4}$ \\
\hline
\end{tabular}

década, com 2003 casos (Tabela 1).

$\mathrm{Na}$ série histórica foi observado aumento de $47,3 \%$ no número total de diagnósticos por DC aguda em todo o território brasileiro entre a primeira década, com 3.088 casos, e a segunda década, com 2.096 casos.

A Unidade Federativa de notificação e de infecção 
original com maior incidência durante o período geral foi o Pará com 2.347 (45,27\%) e 2.076 (40,04\%) casos, respectivamente (Tabela 4$)$.

Tabela 4. Casos confirmados de Doença de Chagas aguda, no Brasil, entre 2001 e 2018, segundo Unidade Federativa de notificação e de infecção.

\begin{tabular}{|c|c|c|}
\hline Unidade Federativa & Notificação & Infecção \\
\hline \multicolumn{3}{|l|}{ Região Norte } \\
\hline Rondônia & 47 & 28 \\
\hline Acre & 41 & 45 \\
\hline Amazonas & 160 & 140 \\
\hline Roraima & 4 & 2 \\
\hline Pará & 2.347 & 2.076 \\
\hline Amapá & 236 & 178 \\
\hline Tocantins & 48 & 37 \\
\hline \multicolumn{3}{|l|}{ Região Nordeste } \\
\hline Maranhão & 75 & 72 \\
\hline Piauí & 165 & 154 \\
\hline Ceará & 57 & 47 \\
\hline Rio Grande do Norte & 256 & 241 \\
\hline Paraíba & 113 & 71 \\
\hline Pernambuco & 316 & 193 \\
\hline Alagoas & 65 & 60 \\
\hline Sergipe & 124 & 100 \\
\hline Bahia & 502 & 465 \\
\hline \multicolumn{3}{|l|}{ Região Sudeste } \\
\hline Minas Gerais & 97 & 102 \\
\hline Espírito Santo & 38 & 15 \\
\hline Rio de Janeiro & 44 & 8 \\
\hline São Paulo & 46 & 19 \\
\hline \multicolumn{3}{|l|}{ Região Sul } \\
\hline Paraná & 31 & 21 \\
\hline Santa Catarina & 74 & 43 \\
\hline Rio Grande do Sul & 186 & 120 \\
\hline \multicolumn{3}{|l|}{ Região Centro-Oeste } \\
\hline Mato Grosso do Sul & 30 & 22 \\
\hline Mato Grosso & 14 & 10 \\
\hline Goiás & 61 & 51 \\
\hline Distrito Federal & 7 & 1 \\
\hline Total & \multicolumn{2}{|c|}{5.184} \\
\hline
\end{tabular}

No grupo da primeira década estudada, o ano de 2003 demonstrou o maior número de diagnósticos clínicos através da apresentação do primeiro sintoma da doença, com 585 casos e, na segunda década, o ano de 2018 com 361 casos (Figura 1).

A forma de contágio da infecção na DC mais frequentemente relatada foi a oral, com $1.978(38,15 \%)$ casos, seguida da vetorial, com $1.834(35,37 \%)$ casos,

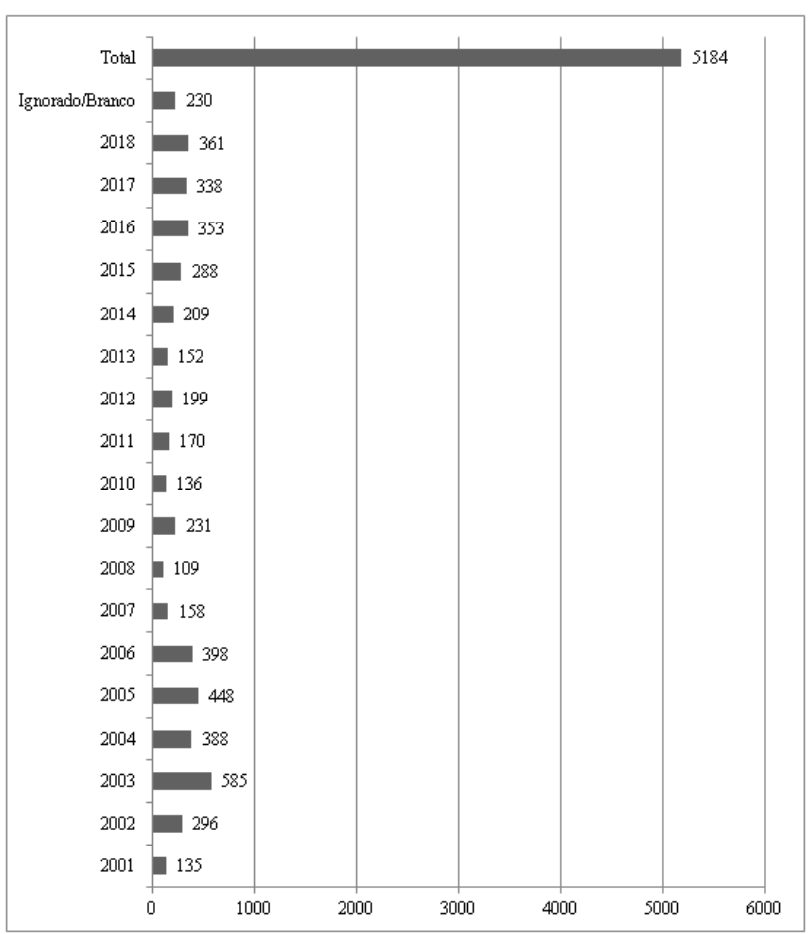

Figura 1. Número de casos confirmados de Doença de Chagas aguda, por ano. durante o período total analisado.

transfusional/acidente de laboratório, com $20(0,38 \%)$ casos e a vertical, com $19(0,36 \%)$ casos. No grupo da primeira década foi observado predomínio de infecção pela forma vetorial, com $1.664(53,92 \%)$ casos e na segunda década, pela forma oral com $1.587(75,35 \%)$ casos (Figura 2).

O local provável para a ocorrência da infecção foi o domicílio em 2.999 (57,85\%) casos, 195 (3,76\%) em laboratórios/outros locais, $32(0,61 \%)$ em unidades de hemoterapia e $1.958(37,77 \%)$ foram ignorados ou deixado em branco (Figura 3).

O critério de confirmação diagnóstica mais utilizado foi o laboratorial com $4.726(91,16 \%)$ casos, seguido pelo clínico-epidemiológico $267(5,15 \%)$ e ainda estavam em investigação $6(0,11 \%)$ casos (Figura 4).

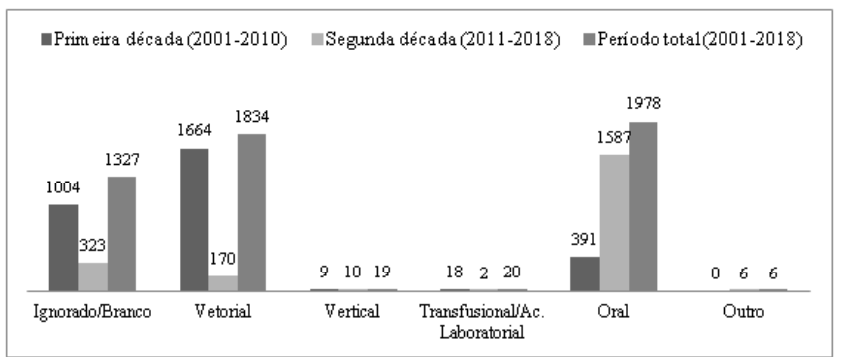

Figura 2. Número de casos confirmados de Doença de Chagas aguda, segundo a forma de transmissão dividida nos seguimentos de década. 


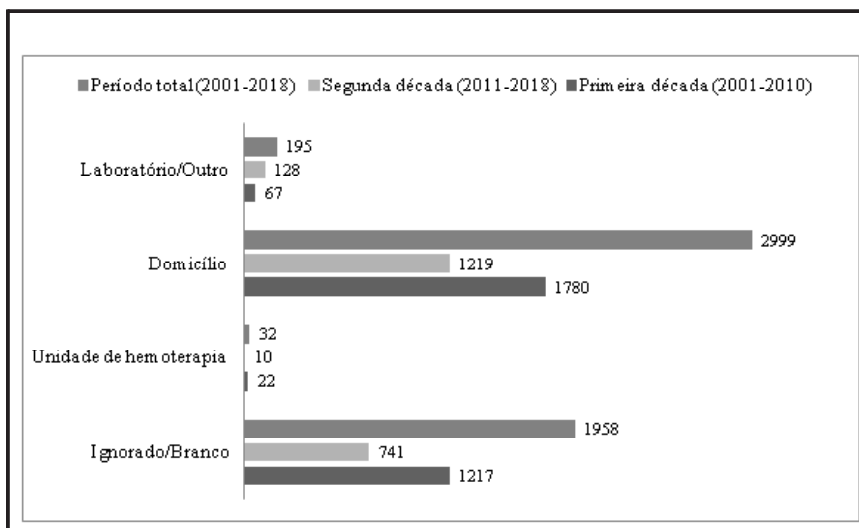

Figura 3. Número de casos confirmados de Doença de Chagas aguda, segundo o local da infecção, dividida nos seguimentos de década.

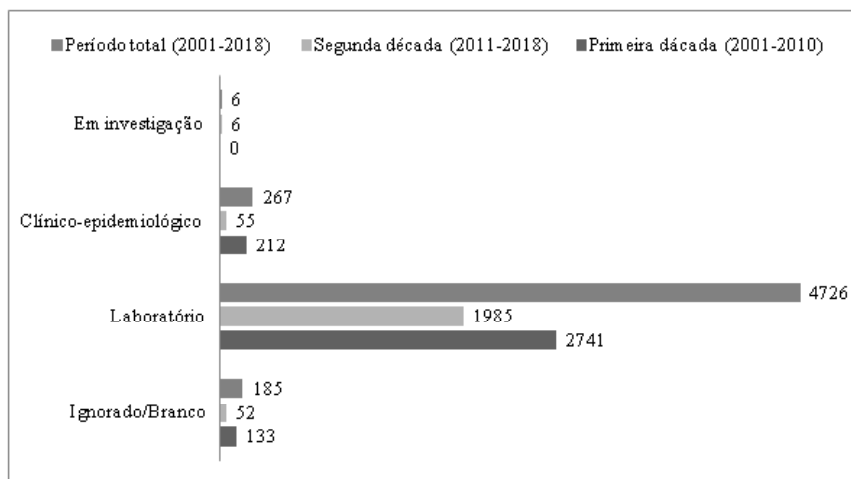

Figura 4. Número de casos confirmados de Doença de Chagas aguda, segundo o critério de confirmação diagnóstica, dividida nos seguimentos de década.

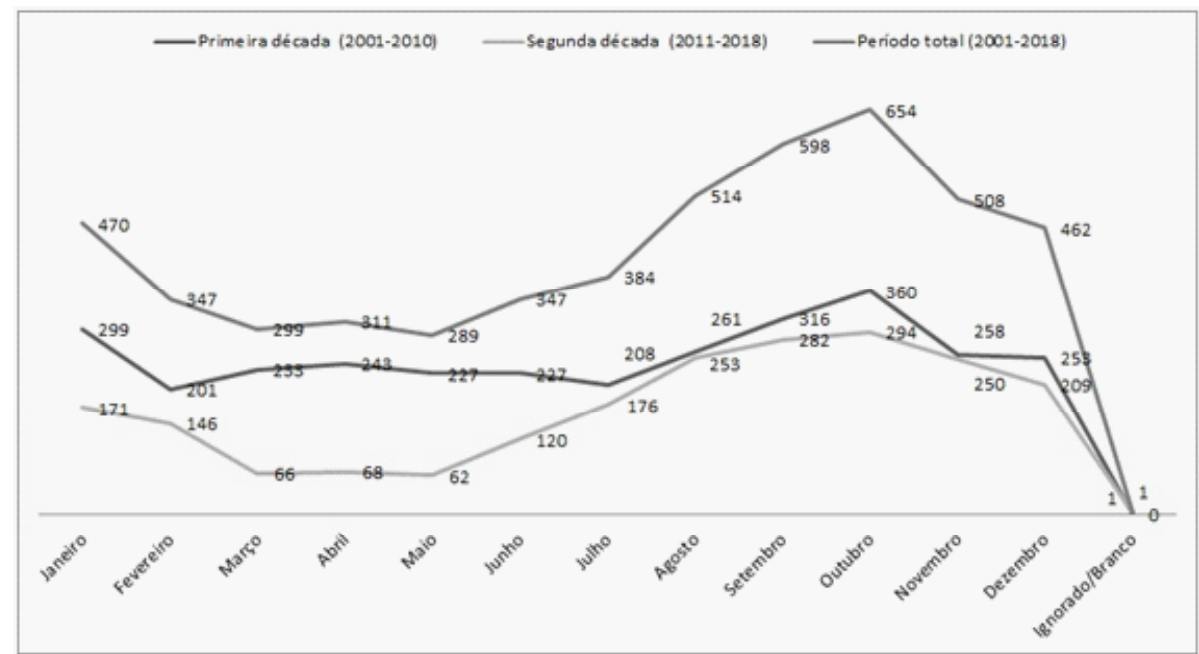

Figura 5. Variação Sazonal do número de casos confirmados de Doença de Chagas aguda no território brasileiro dividida nos seguimentos da primeira e segunda década.

Em relação a evolução clínica desses pacientes, foram registrados $3.510(67,70 \%)$ indivíduos vivos após tratamento, enquanto 134 (2,58\%) evoluíram em óbito pelo agravo notificado durante todo o período estudado (Tabela 5).

Uma exceção do DATASUS foi observada no período entre 2001 e 2006 quanto à variável de evolução clínica, pois o sistema disponibilizou os dados detalhados dos pacientes vivos, que corresponderam a 1.173 $(22,62 \%)$ casos de todos os indivíduos vivos durante

Tabela 5. Formas de evolução clínica da Doença de Chagas aguda, entre 2001 e 2018, no Brasil.

\begin{tabular}{lc}
\hline Evolução clínica & $\mathbf{n}$ \\
\hline Ignorado/Branco & 1.534 \\
Vivo & 3.510 \\
Óbito pelo agravo notificado & 134 \\
Óbito por outra causa & 6 \\
\hline Total & $\mathbf{5 . 1 8 4}$ \\
\hline
\end{tabular}

as duas décadas. Nesse grupo foram registrados 889 $(75,78 \%)$ casos de remissões de manifestação clínica; 151 (2,91\%) negativações das reações sorológicas e 133 $(2,56 \%)$ remissões de parasitemia.

Como se observa na Figura 5, houve variação sazonal no número de diagnósticos por DC no território brasileiro, com maiores índices entre agosto e novembro. O mês de maior incidência diagnóstica foi outubro, com 654 registros, sendo $126,29 \%$ maior do que maio, que foi o mês de menor incidência.

\section{Discussão}

As estimativas mais recentes, segundo o II Consenso Brasileiro em Doença de Chagas, sobre o número de indivíduos infectados pela DC no Brasil variam de 1,9 a 4,6 milhões de pessoas ${ }^{12}$. Em 1996, estimou-se uma prevalência de 1,96 milhão de pessoas infectadas $^{22}$ no país, já em 2006, a Organização Panamericana da Saúde, apresentou novos dados afirmando que 1,9 milhão de brasileiros eram portadores da 
doença ${ }^{16}$, o que reflete a necessidade do país de priorizar a análise cuidadosa sobre esses números ${ }^{15}$.

Em 2014, o primeiro estudo de revisão sistemática e metanálise foi publicado com a intenção de estimar a prevalência da DC no Brasil, contendo publicações entre 1980 a 2012, o qual estimou que havia 4,6 milhões de infectados no país ${ }^{23}$.

Outro estudo publicado em 2015 avaliou o período entre 2000 e 2013, sendo identificado cerca de 1.570 casos notificados por DC aguda em todo o território brasileiro ${ }^{24}$.

A variabilidade dos dados apresentados em cada estudo demonstrou a limitação dos achados e a presença de um silêncio epidemiológico sobre a doença, ou seja, períodos marcados por ausência de registro de notificações oficiais.

No presente estudo, observamos a confirmação de 5.184 casos diagnosticados e notificados de DC aguda no país durante 2001 e 2018, entretanto, acredita-se que esse dado seja maior na prática médica, devido aos casos de subnotificações. Em confirmação dessa teoria, estudos apontam que mais de $80 \%$ dos pacientes infectados com DC nunca tiveram acesso ao diagnóstico ${ }^{12}$, desconhecendo a condição da doença e possível tratamento. Ao mesmo passo que atualizações epidemiológicas realizadas pela Organização PanAmericana de Saúde (OPAS) estimam que cerca de 70 milhões de pessoas vivam em áreas de exposição e estejam em condições suscetíveis de contrair a doença ${ }^{27}$.

No que diz respeito ao perfil epidemiológico da DC, as maiores prevalências verificadas em estudos já publicados foram em: mulheres, maiores de 60 anos, residentes das regiões Nordeste e Sudeste e em áreas mistas, urbana $/ \mathrm{rural}^{23}$. Diferentemente dos achados da literatura, o estudo atual demonstrou que a maior prevalência, nos brasileiros infectados ao longo dessas duas últimas décadas, foi nos indivíduos do sexo masculino, em idade produtiva - adulto jovem entre 40 a 59 anos, pardos e residentes das regiões Norte e Nordeste do país. Estudos mais recentes mostram a presença de uma variação do perfil anteriormente tido como padrão, reafirmando os achados, tais como, os realizados em 2018 com uma população ribeirinha do estado do Pará ${ }^{8}$ e com doadores de sangue no Estado do Piaui ${ }^{29}$

A manifestação mais comum da DC é a forma crônica, todavia, os dados epidemiológicos sobre essa forma ainda é de difícil magnitude, uma vez que apenas as formas agudas são notificadas compulsoriamente pelo SINAN e muitos pacientes crônicos terão desconhecimento da existência da mesma até que venham a desenvolver complicações esperadas para o curso clínico $^{12}$, considerando que cerca de 30 a $40 \%$ dos infectados desenvolverão lesões cardíacas e digestivas severas em um período de 5 a 20 anos após a infecção ${ }^{6}$. O presente estudo evidenciou uma incidência considerada baixa para o período de análise de duas décadas, entretanto, justificada pelas notificações serem referentes a DC aguda, podendo ser enquadrado, segundo estudos, na porcentagem de $10-20 \%$ dos casos que são de fato notificados ${ }^{12}$.

As formas de transmissão de DC podem ser por transmissão oral, vetorial, acidente laboratorial ou vertical. Segundo pesquisa que avaliou o período de 2000 a 2013, foi demonstrado que $70 \%$ dos 1.570 infectados por DC aguda ocorreram pela forma ora ${ }^{24}$, principalmente, após o surto da transmissão pela cana de açúcar em Santa Catarina, em 2005. Não obstante, em estudo recente realizado em 2018, observou-se ainda a correlação da transmissão oral, através do consumo de caldo de cana, com o surto confirmado de 21 casos de DC em quatro municípios do Estado do Rio Grande do Norte $^{30}$.

Entretanto, apesar da ocorrência em menor proporção, pesquisas realizadas entre 2008 e 2012, apontaram que $10 \%$ de todos os registros do período ocorreram por transmissão vetorial, contra $64 \%$ por transmissão oral e $13 \%$ de forma não identificada ${ }^{25}$. Além disso, segundo pesquisa realizada em $2018^{31}$, a transmissão oral parece ocasionar manifestações clínicas mais graves e maior mortalidade do que a transmissão vetorial.

A transmissão vertical, conforme comprovações científicas divulgadas em 2020, depende de fatores inerentes ao parasita e ao hospedeiro. A fisiopatologia do mecanismo é explicada pelas formas tripomastigotas adentrarem no estroma das vilosidades, dos troncos vilosos ou da placenta corial da gestante, onde passam a parasitar os macrófagos da placenta, se transformam e após ruptura da célula, podem parasitar outros macrófagos, atingindo o feto ${ }^{32}$. No entanto, conforme a literatura aponta, o risco de transmissão por via transplacentária entre as gestantes brasileiras infectadas pela DC gira em torno de $1 \%$, sendo que em outras áreas da América Latina, este percentual pode aumentar até $12 \%{ }^{12}$. Sendo possível comprovar que as assertivas reforçam com os achados desde estudo acerca da baixa porcentagem de infecção por via vertical.

Não obstante, o atual estudo evidenciou uma maior prevalência da forma oral, corroborando com os achados da literatura e a explicação epidemiológica para essa ser a forma de transmissão mais representativa no território brasileiro. Entretanto, a forma vetorial é a segunda forma mais registrada e esse achado deve receber uma análise crítica já que a realidade brasileira ainda apresenta riscos relacionados a esse modo de transmissão, tais como, a existência de espécies de triatomíneos autóctones com elevada capacidade de se colonizar e invadir unidades domiciliares $^{26}$ e a persistência de focos de Triatoma infestans como relatado nos anos de 2012 e 2016 nos estados da Bahia e Rio Grande do Sul ${ }^{24}$. 
É predominante a contaminação em pacientes com maior exposição aos triatomíneos, atribuída, de forma multifatorial, às moradias precárias e à degradação do meio ambiente, conforme demonstra pesquisa realizada no município de Abaetetuba e Barcarena, Pará ${ }^{33}$.

Estudos realizados entre 2016 e 2019, evidenciaram a associação da DC com os indicadores sociais de baixa qualidade, onde foi possível observar um predomínio de infecção em indivíduos de baixa escolaridade e em situação de maior vulnerabilidade social. Além de demonstrar a associação entre a presença de triatomíneos e as mudanças ambientais oriundas da ocupação humana ${ }^{34,35}$ especialmente o desmatamento, visto que impulsiona a migração dos vetores para novas áreas, contribuindo para que os animais silvestres, que são fontes de alimentos para o repasto sanguíneo dos triatomíneos, migrem para áreas domésticas. Em consequência das alterações ecológicas, há a movimentação dos barbeiros para estes ambientes peridomésticos, levando os vetores a invadir o ambiente doméstico $^{36}$ e ocupar as proximidades dos locais de manipulação de alimentos ${ }^{37}$.

Dados de estudo encontrados na literatura comprovaram registros de DC aguda na maioria dos estados brasileiros, com maior distribuição, cerca de $90 \%$, na região Norte, sendo o estado do Pará responsável por $84 \%$ dos $\operatorname{casos}^{25}$. Da mesma forma, o estudo que avaliou o período entre 2000 e 2013 corroborou com esses achados, uma vez que, dos 1.570 casos confirmados registrados, a maioria era oriunda da região Norte, seguida pela Nordeste, sendo o estado do Pará o responsável por $75 \%$ dos casos de todo o país ${ }^{24}$. Segundo o Boletim Epidemiológico de 2018, foram notificados 4.685 indivíduos suspeitos com DC aguda, e destes, 380 foram confirmados $(8,1 \%)$, sendo a região Norte a de maior proporção de casos no ano analisado $(92,1 \%)$, com uma incidência de 1,93 casos/100 mil habitantes e dos casos totais confirmados, $76,3 \%$ residiam do estado do Pará ${ }^{38}$.

O achado epidemiológico do presente estudo também vai de encontro com os demais registros literários, uma vez que a maior incidência de infectados nos últimos 18 anos ocorreu na Região Norte e Nordeste, também tendo como o estado de maior destaque em número de infectados, o Pará, correspondendo a 40,04\% de todos os registros. Vale ressaltar ainda, que durante esse período cerca de 863 casos não obtiveram registros quanto a UF responsável pela infecção.

A limitação dessa pesquisa epidemiológica faz referência a confiabilidade restringida pelo nível de acurácia e dependência do fornecimento das notificações apresentadas e preenchidas pelo banco de dados do DATASUS. As observações obtidas neste estudo podem não representar toda a magnitude da DC no território brasileiro. Entretanto, mesmo com essas limitações, o presente estudo apresenta de forma ampla e atualizada dados para análise epidemiológica da DC ao longo das duas últimas décadas no país, possibilitando discriminar e estratificar o tipo de perfil do paciente infectado e assim desenvolver com eficácia políticas voltadas ao diagnóstico e tratamento precoce ao paciente acometido.

É indicado a realização da rotina de avaliações para os sobreviventes da doença, conforme preconizado no II Consenso Brasileiro em Doença de Chagas, para a verificação de cura e para o acompanhamento em longo prazo do caso tratado. Além disso, é necessário efetivar a prevenção terciária a esses indivíduos, reabilitando os pacientes que desenvolveram possíveis complicações do quadro agudo ou tenham atribuído a forma crônica da doença.

\section{Conclusão}

Os dados epidemiológicos de pacientes infectados e notificados com DC no território brasileiro, entre o período de 2001 e 2018, demonstrou predomínio em adultos jovens do sexo masculino, pardos e moradores das regiões economicamente menos subsidiadas do país.

Considerando que os resultados demonstraram uma maior recorrência entre esse perfil epidemiológico, sugere-se um maior investimento e atenção da equipe multidisciplinar e dos gestores a esse grupo populacional.

São necessárias medidas preventivas e de monitorização epidemiológica dos casos confirmados e suspeitos, nas formas crônicas e agudas, a fim de que haja controle de possíveis focos vetoriais e outras formas de infecção.

\section{Referências}

1. Dias JCP, Amato Neto V, Luna EJA. Mecanismos alternativos de transmissão do Trypanosoma cruzi no Brasil e sugestões para sua prevenção. Rev Soc Bras Med Trop. 2011; 44(3): 375-9.

2. Rassi Jr. A, Rassi A, Rezende JM. American trypanosomiasis (Chagas disease). Infect Dis Clin North Am. 2012; 26(2): 275-91.

3. Santos F, Lyra MAM, Alvez LDS, et al. Pesquisa, desenvolvimento e inovação para o controle das doenças negligências. Rev Ciênc Farm Bas Aplic. 2012; 33(1): 37-47.

4. Dias JCP. Human chagas disease and migration in the context of globalization: some particular aspects. J Trop Med. 2013:789758.

5. Lana M, Tafuri WL. Trypanosoma cruzi e doença de Chagas. In: Neves DP, editor. Parasitologia humana. 11 ed. São Paulo: Atheneu; 2004. 85-108.

6. Rassi A Jr, Rassi A, Marin-Neto JA. Doença de Chagas. Lancet. 2010; 375 (9723): 1388-402. 
7. World Health Organization. Research priorities for Chagas disease, human African trypanosomiasis and leishmaniasis. WHO: technical report of the TDR Disease Reference Group on Chagas Disease, Human African Trypanosomiasis and Leishmaniasis. Geneva: World Health Organization; 2012. (WHO Technical Report Series, 975).

8. World Health Organization. Sustaining the drive to overcome the global impact of neglected tropical diseases: second WHO report in neglected tropical diseases. Geneva: World Health Organization; 2013.

9. Coura JR, Viñas PA, Junqueira AC. Ecoepidemiology, short history and control of Chagas disease in the endemic countries and the new challenge for non-endemic countries. Mem Inst Oswaldo Cruz. 2014;109(7):856-62.

10. Prata A. Clinical and epidemiological aspects of Chagas disease. Lancet Infect Dis. 2001 Sep;1(2):92-100.

11. Coura JR. The main sceneries of Chagas disease transmission. The vectors, blood and oral transmissions: a comprehensive review. Mem Inst Oswaldo Cruz. 2015;110(3):277-82

12. Ministério da Saúde (Brasil). Secretaria de Vigilância em Saúde. II Consenso Brasileiro em Doença de Chagas, 2015. Epidemiol Serv Saúde 2016; 25; Epub 30-Jun-2016

13. Organização Mundial da Saúde. Pan American Health Organization (PAHO). General information Chagas disease. 2016. Available at: http:// www.paho.org/hq/index.php?option $=$ com content $\& v i e w=$ article \&id $=5856$ \&Itemid=41506\&lang=en Acesso em: 16 junho 2020.

14. World Health Organization. Chagas disease in Latin America: an epidemiological update based on 2010 estimates. Wkly Epidemiol Rec. 2015;90(6):33-44

15. Saúde de A a Z: Doença de Chagas. 2018. Disponível em: < https:// antigo.saude.gov.br/saude-de-a-z/doenca-de-chagas $>$. Acesso em: 16 junho 2020

16. Pan American Health Organization. Estimación cuantitativa de la enfermedad de Chagas en las Americas. Washington: Pan American Health Organization; 2006

17. Bern C, Montgomery SP. An estimate of the burden of Chagas disease in the United States. Clin Infect Dis. 2009;1:49(5):e52-4

18. Centers for Disease Control and Prevention. Center for Global Health. Division of Parasitic Diseases and Malaria. Chagas disease in the Americas: 2013. Atlanta: Centers for Disease Control and Prevention; 2013.

19. Hotez PJ, Dumonteil E, Betancourt Cravioto M, Bottazzi ME, TapiaConyer R, Meymandi S, et al. An unfolding tragedy of Chagas disease in North America. PLoS Negl Trop Dis. 2013;7(10):e2300.

20. European Centre for Disease Prevention and Control. Assessing the burden of key infectious diseases affecting migrant populations in the EU/ EEA: Technical Report. Stockholm: ECDC; 2014.

21. Requena-Méndez A, Aldasoro E, Lazzari E, Sicuri E, Brown M, Moore DA, et al. Prevalence of Chagas disease in Latin-American migrants living in Europe: a systematic review and meta-analysis. PLoS Negl Trop Dis. 2015 Feb;9(2):e0003540.

22. Akhavan D. Análise de Custo-Efetividade do Programa de Controle da Doença de Chagas no Brasil: Relatório Final. Brasília: Organização PanAmericana de Saúde; 1998

23. Martins-Melo FR, Ramos Júnior AN, Alencar $\mathrm{CH}$, Heukelbach J. Prevalence of Chagas disease in Brazil: a systematic review and metaanalysis. Acta Trop. 2014;130:167-74.

24. Ministério da Saúde (Brasil). Secretaria de Vigilância em Saúde. Doença de Chagas aguda no Brasil: série histórica de 2000 a 2013. Bol Epidemiol. 2015;46(21):1-9.

25. Ministério da Saúde (Brasil). Secretaria de Vigilância em Saúde. Coordenação-Geral de Desenvolvimento da Epidemiologia em Serviços. Guia de vigilância em saúde [Internet]. Brasília: Ministério da Saúde; 2016. 773 p. Disponível em: http://portalarquivos2. saude.gov.br/images/pdf/2016/ agosto/25/GVS-online.pdf Acesso em: 16 junho 2020.

26. Silveira AC, Dias JCP. O controle da transmissão vetorial. Rev Soc Bras Med Trop 2011;44(2):52-63.
27. Organização Pan-Americana da Saúde-PAHO|Chagas disease.https:// www.paho.org/hq/ index.php?option=com topics\&view=article\&id=10\&I temid=40743\&lang=en. Accessed November 21, 2019.

28. Cardoso LP, Paiva TR, Nogueira LMV, Guimarães RJPS, Rodrigues ILA, André SR. Distribuição espacial da doença de Chagas e sua correlação com os serviços de saúde. Rev Esc Enferm USP. 2020;54: e03565. Epub 26Jun-2020.

29. Santana MP, Santos RS, Almeida AS. Prevalência da Doença de Chagas entre Doadores de Sangue do Estado do Piaui, Brasil, no período de 2004 a 2013. Cad Saúde Pública. 2018;34(2):e00123716

30. Vargas A, Malta JMAS, Costa VM, Cláudio LDG, Alves RV, Cordeiro GS, Aguiar LMA, Percio J. (2018). Investigação de Surto de Doença de Chagas Aguda na Região Extraamazônica, Rio Grande do Norte, Brasil. Cad Saúde Pública. 2016; 34(1). e00006517

31. Pérez-Molina JA, Molina I. Chagas disease. Lancet 2018;391(10115):82-94.

32. Avila WS, Alexandre ERG, Castro ML, Lucena AJG, Marques-Santos C, Freire CMV, Rossi EG, Campanharo FF, Rivera IR, Costa MENC, Rivera MAM, Carvalho RCM, Abzaid A, Moron AF, Ramos AIO, Albuquerque CJM, Feio CMA, Born D, Silva FB, ... Leal TCAT. (2020). Posicionamento da Sociedade Brasileira de Cardiologia para Gravidez e Planejamento Familiar na Mulher Portadora de Cardiopatia - 2020. Arq Bras Cardio. 2020;114(5), 849-942.

33. Guimarães RJPS, Sousa Junior AS, Pinheiro AF, Dias RS, Dias FA, Neves ALL, et al. Geoprocessamento aplicado à análise ecoepidemiológica da doença de Chagas nos municípios de Abaetetuba e Barcarena, no Estado do Pará, Brasil, entre 2000-2011. Rev SELPER. 2012;34(2):30- 5

34. Vianna EN, Guimarães RJPS, Souza CR, Gorla D, Diotaiu/ti L. Chagas disease ecoepidemiology and environmental changes in northern Minas Gerais state, Brazil. Mem Inst Osw Cruz. 2017;112(11):760-8.

35. Dias JVL, Queiroz DRM, Martins HR, Gorla DE, Pires HHR, Diotaiuti L. Spatial distribution of triatomines in domiciles of an urban area of the Brazilian Southeast Region. Mem Inst Osw Cruz. 2016;111(1):43-50.

36. Guhl F. Geographical distribution of Chagas disease. In: Telleria J, Tibayrenc M, editors. American Trypanosomiasis Chagas Disease. Cambridge: Academic Press; 2017. 89-112.

37. Echeverria LE, Morillo CA. American Trypanosomiasis (Chagas Disease). Infect Dis Clin N Am. 2019;33(1):119-34.

38. Ministério da Saúde (Brasil). Secretaria de Vigilância em Saúde. Boletim Epidemiológico - Panorama da Doença de Chagas no Brasil, 2019. Epidemiol Serv Saúde. 2019;50(36). Epub Nov-2019. 